\title{
Pressão arterial e indicadores de função vascular de corredores com diferentes níveis de desempenho no teste cardiopulmonar
}

http://dx.doi.org/10.11606/1807-5509201700040747

\author{
Edna Oliveira SILVA* \\ Anselmo José PEREZ* \\ Luiz Aparecido BORTOLOTTO** \\ Wellington LUNZ* \\ José Geraldo MILL* \\ Luciana CARLETTI*
}

*Centro de Educação

Física e Desportos,

Universidade Federal

do Espírito Santo,

Vitória, ES, Brasil.

${ }^{* *}$ Faculdade

de Medicina,

Universidade de São

Paulo, São Paulo, SP

Brasil.

\section{Resumo}

0 objetivo deste estudo foi comparar os valores de pressão arterial sistólica e diastólica periférica (PASp e PADp) e central (PASc e PADc) e o índice de função vascular em corredores de rua de diferentes desempenhos. A amostra foi composta por 48 participantes, 20-40 anos, 32 corredores de rua com diferentes níveis de desempenho, e 16 indivíduos sedentários. Avaliou-se a aptidão cardiorrespiratória e a velocidade máxima de corrida, através do teste cardiopulmonar de exercício (TCPE). A pressão central e periférica foi investigada por tonometria de aplanação e a velocidade da onda de pulso (VOP) pelo equipamento Complior. 0 ecocardiograma foi usado para avaliar a estrutura cardíaca. ANOVA de uma via foi utilizada considerando $p<0,05$. A ANCOVA foi usada para ajustar fatores de confusão. Corredores com desempenho superior apresentaram menor PASp que os demais grupos ( $120 \pm 7$ vs $127 \pm 8$ vs $130 \pm 8 \mathrm{mmHg}$ ). A duração da diástole foi maior nos corredores com desempenho superior ( $845 \pm 92 \mathrm{~ms}$ ) em relação aos corredores com desempenho inferior ( $786 \pm 174 \mathrm{~ms}$ e controle $(641 \pm 128 \mathrm{~ms}) .0$ índice de amplificação (Al) não foi diferente entre os grupos ( $109 \pm 21 \% ; 109$ $\pm 11 \% ; 110 \pm 1 \%)$ assim como a VOP $(7,1 \pm 1 \mathrm{~ms} .7,6 \pm 1.1 \mathrm{~ms} .8,0 \pm 1,1 \mathrm{~ms})$. Corredores com desempenho superior apresentaram maior massa do ventrículo esquerdo (MVE $\left.\mathrm{g} / \mathrm{m}^{2}\right)$ em relação aos demais $(116 \pm 12$ $\mathrm{g} / \mathrm{m}^{2}$ vs $100 \pm 18 \mathrm{~g} / \mathrm{m}^{2}$ vs $\left.86 \pm 13 \mathrm{~g} / \mathrm{m}^{2}\right)$, e menor débito cardíaco em comparação ao grupo controle $(4,8 \pm 1 \mathrm{I} /$ min. vs $6,0 \pm 1 \mathrm{l} / \mathrm{min}$ ). Portanto corredores com desempenho superior apresentaram maior MVE $\mathrm{g} / \mathrm{m}^{2}$, menor débito cardíaco, menor PASc e PASp, e melhor duração da diástole que seus pares sedentários, mas não apresentam melhores indicadores de função vascular na mesma comparação.

Palavras-Chave: Exercício Aeróbio; Função Vascular; Rigidez Arterial; Análise da Onda de Pulso.

\section{Introdução}

Os benefícios do exercício aeróbio regular sobre o sistema cardiovascular têm sido bem estabelecidos em humanos ${ }^{1}$. Entretanto existe uma discussão sobre o efeito crônico do exercício aeróbio mais intenso na saúde cardiovascular ${ }^{2,3}$. A preocupação em estudar os efeitos adversos causados pela prática do exercício aeróbio extenuante se justifica, pelo fato de que muitos indivíduos iniciam a prática de corridas mais vigorosas muitas vezes na meia-idade, o que incorre no aumento da chance de apresentarem fatores de risco cardiovasculares ${ }^{4}$ e ainda em decorrência do treinamento intenso realizado por atletas de endurance, visando à busca do melhor desempenho esportivo, expondo o coração a intensas sobrecargas ao longo de meses ou anos. Uma exposição frequente de sobrecarga resulta em alteraçôes no automatismo cardíaco, como bradicardia de repouso, e alteração de condução átrio ventricular, despolarização e repolarização ventricular ${ }^{5,6}$.

Os ajustes estruturais do coração também são marcantes, podendo levar a aumento de até $85 \%$ na massa do ventrículo esquerdo ${ }^{7}$. Apesar dessas alteraçôes funcionais e estruturais serem devidamente documentadas, ainda são desconhecidos os seus limites de normalidade e suas consequências em longo prazo. Dessa forma a literatura apresenta que a prática 
de exercícios aeróbios pode estabelecer uma relação dose-resposta, onde a inatividade é prejudicial, mas o exercício excessivo pode ser igualmente prejudicial. Entretanto ainda não se sabe qual o limite de estresse para o surgimento dos efeitos prejudiciais em praticantes de treinamento de endurance ${ }^{3,8}$. Dentre alguns prejuízos encontrou-se maior rigidez arterial em maratonistas ${ }^{9}$, aumento do escore de cálcio coronário $^{10}$, e função sistólica do ventrículo direito reduzida em maratonistas amadores, causada por aumento na inflamação e diminuiçáo no fluxo de sangue $^{11}$. E ainda, maior pressão de pulso periférica e maior pressão sistólica, diastólica em indivíduos treinados, mas não maratonistas ${ }^{12}$.

Em atletas de endurance encontrou-se pressão central aórtica inalterada, sendo esse efeito não necessariamente benéfico, mas também não prejudicial, por outro lado a viabilidade subendocárdica apresentou se aumentada ${ }^{13}$, assim como uma reduçáo da VOP e aumento da complacência central ${ }^{14}$ Redução significante na VOP central e periférica, e menor índice de incremento corrigido pela frequência cardíaca foi encontrada em não maratonistas (AIx@75) ${ }^{15}$. Menor rigidez arterial e menor valor de PAS periférica, em um grupo com maior tempo de pratica de corrida ${ }^{16}$. Em um estudo

\section{Método}

\section{Seleção da amostra}

Participaram desse estudo 32 corredores do sexo masculino entre 20 e 40 anos, recrutados em provas de corridas de rua, do circuito capixaba, e 16 indivíduos controle do sexo masculino que foram recrutados por meio de convite à comunidade estando isentos de prática de qualquer exercício físico regular nos últimos seis meses, totalizando uma amostra de 48 voluntários, todos sem uso de medicação, assintomáticos e sem evidência de doença cardiovascular após avaliação clínica e laboratorial. A avaliação laboratorial foi realizada após 12 horas em jejum, aproximadamente às 7 horas da manhã, em uma única visita ao Hospital Universitário na clínica de investigaçáo cardiovascular (CIC) do Centro de Ciências da Saúde da Universidade Federal do Espírito Santo, com exceção para o teste cardiopulmonar, que foi realizado no período da tarde no Laboratório de Fisiologia do Exercício de coorte observaram que sujeitos com menor aptidão aeróbia determinada pelo teste ergométrico máximo, tinham risco relativo de 1,5 para a incidência de hipertensão arterial em relação aos sujeitos com maior aptidão aeróbia ${ }^{17}$. Contudo as evidências são bem convincentes a respeito das melhorias na função cardiovascular com o condicionamento aeróbio. O condicionamento cardiorrespiratório é um dos componentes da aptidáo física diretamente associada aos níveis de saúde e qualidade de vida. $\mathrm{Na}$ função vascular o efeito crônico do treinamento aeróbio resulta diretamente no aumento no volume de ejeção, redução da frequência cardíaca, aumento do débito cardíaco, aumento na capacidade de captação de oxigênio e redução da pressão artérial ${ }^{18}$. Dessa forma o objetivo desse estudo foi comparar as variáveis da hemodinâmica central e periférica através de um método não invasivo, de corredores classificados por diferentes níveis de desempenho no teste cardiopulmonar (velocidade de corrida máxima). A hipótese a ser testada é a de que, em condição de repouso, corredores classificados com desempenho superior, mas não maratonistas, apresentam melhor função vascular e função cardíaca em relação a corredores com desempenho inferior e grupo controle.
(LAFEX). Foram observados um período de pelo menos $24 \mathrm{~h}$ entre a última sessão de treinamento, e a aplicação do teste cardiopulmonar. A avaliação clínica incluiu cuidadosa anamnese para obtenção de dados, como idade, sexo, etnia, e presença ou não dos critérios de exclusão. A etnia foi registrada conforme referida pelos próprios indivíduos, que foram classificados como brancos. Os critérios para o recrutamento foram: a) possuir idade entre 20 e 40 anos, b) participar de corrida de rua há pelo menos 6 meses e treinar de 3 a 7 vezes por semana, c) náo apresentar problemas ortopédicos e/ou de doença cardiopulmonar ou metabólica; d) não ser fumante ou caracterizado com qualquer outro tipo de dependência química; e) não fazer uso de medicamentos ergogênicos ou qualquer outro tipo de medicamento que poderia influenciar os resultados dos exames. Todos assinaram termo de consentimento livre e esclarecido. O estudo foi aprovado pelo comitê de ética em pesquisa da 
Universidade Federal do Espírito Santo (CEP/UFES, 009/2010).

Do grupo de corredores $(\mathrm{n}=32), 16$ foram considerados com nível de desempenho superior (GSup), e 16 com nível de desempenho inferior (GInf), de acordo com o percentil 50 da amostra (p50). A classificação do nível cardiorrespiratório foi realizada pelo desempenho no teste cardiopulmonar por meio da velocidade máxima atingida.

\section{Cálculo amostral}

O cálculo amostral foi desenvolvido pelas diferenças de valores de pressão arterial, central e periférica, entre os grupos de desempenho, obtidos em estudo prévio desenvolvido ${ }^{19}$. Utilizando a diferença de 9 $\mathrm{mmHg}$ (entre a pressão arterial sistólica, PAS - central do grupo superior vs inferior), e considerando um desvio-padrão de $9 \mathrm{mmHg}$, chegou-se ao cálculo de 16 indivíduos, de acordo com a fórmula apresentada na literatura ${ }^{20}$. O cálculo para a pressão arterial diastólica (PAD) e pressão arterial média (PAM) fornecia um número menor de indivíduos, sendo assim, optou-se pela PAS.

\section{Medidas antropométricas}

A massa corporal foi medida utilizando uma balança digital (Toledo, São Paulo, Brasil) com precisão de 0,01 kg; a estatura, por meio de um estadiômetro Seca com precisão de $1 \mathrm{~mm}$ (modelo 216, Santa Catarina, Brasil), através dos quais foi calculado o índice de massa corporal (IMC). Os indivíduos vestidos com camisetas e calçôes leves.

\section{Teste Cardiopulmonar de Exercício (TCPE)}

O Teste Cardiopulmonar de Exercício (TCPE) foi realizado em uma sala silenciosa mantida a temperatura ambiente entre 22 e $24^{\circ} \mathrm{C}$, e acompanhado por um cardiologista, que lia os registros eletrocardiográficos e aferia a pressão arterial, para garantir a segurança do teste. Os indivíduos se dirigiam para a esteira ergométrica (Inbrasport, modelo Super ATL, Porto Alegre, Brasil), onde eram equipados com uma máscara facial de silicone, adaptada ao tamanho do rosto e conectada ao pneumotacômetro, para medida do fluxo de ar e análise dos gases expirados. Os indivíduos eram orientados sobre os procedimentos de realização do teste, que incluíam: permanecer respirando normalmente, não usar como apoio o corrimão da esteira durante a caminhada ou corrida, a não ser em caso de necessidade e ao final do teste; solicitar a interrupção, em caso de fadiga, ou desconforto que impossibilitasse a continuidade.

O teste foi realizado seguindo um protocolo de rampa individualizado, objetivando uma duração entre 10 e 12 minutos. A inclinação da esteira permanecia constante (1\%) e a velocidade inicial de $6 \mathrm{~km} / \mathrm{h}$ era incrementada a cada 15 segundos, variando em uma taxa de 0,6 a $1,3 \mathrm{~km} / \mathrm{min}$, de acordo com a velocidade final estipulada. As variáveis ventilatórias foram mensuradas utilizando o analisador metabólico de gases (modelo Córtex Metamax 3B, Alemanha), com coleta respiração a respiraçáo, e em seguida calculadas as médias a cada 10 segundos, sendo analisados com a ajuda do programa Metasoft. A unidade do Cortex foi calibrada pelo método de circuito fechado, através de gás de calibração (cilindro de $16 \% \mathrm{O}_{2}$ e $5 \% \mathrm{CO}_{2}$ original, atestado pelo fornecedor). A cada teste era executada uma nova calibragem do sistema.

$\mathrm{OVO}_{2 \text { máx. }}$ era aceito quando os seguintes critérios eram contemplados ${ }^{21}$ : a) exaustão ou inabilidade para manter a velocidade requerida; b) razáo de taxa respiratória superior a 1,15; c) frequência cardíaca (FC) máxima de pelo menos $90 \%$ da FC máxima estimada (220 - idade). A FC foi registrada pelo ECG durante todo o teste e acompanhada até o terceiro minuto da recuperação. Ao final do teste a velocidade era reduzida de 5 a $4 \mathrm{~km} / \mathrm{h}$ durante um minuto, seguido por mais um minuto de recuperação passiva.

Variáveis coletadas no teste cardiopulmonar de exercício (TCPE) - Os seguintes parâmetros do teste cardiopulmonar foram coletados para comparação entre os grupos: consumo máximo de oxigênio $\left(\mathrm{VO}_{2 \text { máx. }}\right)$ - média dos valores do $\mathrm{VO}_{2}$ coletados ao final do teste, em uma amostragem de 30 segundos, velocidade máxima (Vmáx.) e submáxima (VLAV) - velocidade máxima atingida no momento da identificação do $\mathrm{VO}_{2 \text { máx }}$.

\section{Pressáo arterial de repouso}

A pressão arterial (PA) e a FC foram mensuradas com um aparelho oscilométrico automático (Omron 705CP, Intelissense, Japão) com os pacientes sentados após $5 \mathrm{~min}$ de repouso em uma sala silenciosa com temperatura controladas $\left(22-24^{\circ} \mathrm{C}\right)$. Três medidas foram realizadas com um intervalo de 2 minutos e a média das duas menores mediçóes foi utilizada para análise de dados. 


\section{Análise da onda de pulso}

A análise da onda de pulso foi projetada a partir da técnica de tonometria de aplanação, com o equipamento SphygmoCor ${ }^{\bullet}$ (ArtCor Medical Inc., Sydney, Austrália) para a obtenção da reconstituição da forma indireta, através de algoritmo matemático, da pressão arterial central. Os índices analisados a partir do contorno das ondas de pressáo anterógradas e retrógradas foram obtidos por uma função de transferência previamente validada após calibração com a pressão arterial sistólica e diastólica braquial. A PA braquial foi medida com aparelho oscilométrico automático previamente validado e com certificação de calibração do fabricante (Omron $705 \mathrm{CP}$, Intelissense, Japão). Neste estudo, os principais indicadores hemodinâmicos periféricos analisados a partir da artéria radial foram: PA sistólica periférica (PASp) e diastólica periférica (PADp, Pressão de pulso periférica (PPp) - definida como a diferença entre a PASr e PADr. Os indicadores da hemodinâmica central a partir da artéria aorta foram: FC- frequência cardíaca, pressão sistólica central (PASc) e diastólica (PADc); Pressão de pulso central (PPc), AI- índice de incremento, SERV- viabilidade subendocárdica, DD- duração da diástole. $\mathrm{O}$ exame foi realizado com o indivíduo na posição supina após cinco minutos em repouso. A PA sistólica e PA diastólica da artéria braquial direita foi medida a fim de calibrar o aparelho. Em seguida, posicionavase o tonômetro sobre o ponto de maior pulsação da artéria radial direita para se obter a onda de pressão radial. $\mathrm{O}$ exame foi considerado válido e de boa qualidade quando apresentava o Índice de Operação (operator índex) superior a 80.

\section{Velocidade da onda de pulso}

A medida da VOP foi obtida automaticamente pelo registro de ondas de pulso simultâneas captadas por sensores externos sobre dois pontos conhecidos da árvore arterial (carótida e femoral) e calculada como a distância entre os dois pontos de medida dividida pelo tempo percorrido entre os mesmos, fornecido pelo software do aparelho. $\mathrm{O}$ aparelho Complior ${ }^{\circ}$ (Gonesse, França) foi empregado para a obtenção das medidas. A VOP foi avaliada no segmento arterial carótidafemoral, representando a medida do trânsito da onda de pulso pela aorta. Para obter o valor da VOP de cada avaliado, foram selecionadas dez curvas, com boa qualidade, sendo, então, a média calculada. As curvas foram adquiridas após repouso de cinco minutos com os indivíduos na posição supina.

Os procedimentos de tonometria de aplanação e da VOP foram realizados por uma técnica de enfermagem, treinada e certificada ao longo de todo o estudo. Sendo que a mesma não possuía conhecimento sobre os níveis de desempenho dos indivíduos avaliados.

\section{Ecocardiografia com Doppler}

O ecocardiograma transtorácico foi realizado segundo padronização feita no Projeto ELSA (Estudo Longitudinal de Saúde do Adulto) ${ }^{22}$ em plataforma multiuso (Toshiba Aplio XG, Japão). Foram coletados dados da estrutura cardíaca incluindo cálculo da massa do ventrículo esquerdo, diâmetro de cavidades, diâmetro de aorta, Doppler transmitral e Doppler tissular. Uma análise preliminar da hipertrofia de ventrículo esquerdo (HVE) foi realizada de acordo com a medida do septo interventricular conforme as tabelas do American Heart Association. A massa ventricular esquerda (MVE) foi corrigida para a área de superfície corporal estimada ${ }^{23}$. E altura elevada a potência de $2,7^{24}$. Foram obtidas imagens nos cortes nas janelas paraesternal longitudinal e transversal, apical de 4 câmaras, 2 câmaras e 5 câmaras. As medidas da fração de ejeção do ventrículo esquerdo, espessura das paredes e massa ventricular esquerda foram realizadas pelo eco modo-M. Fração de ejeção normal foi considerada $>0,50$.

\section{Análise estatística}

Os dados são apresentados como média \pm desvio padrão e mediana, quando necessário. A normalidade dos dados foi testada com o teste Shapiro Wilk. Para as variáveis que apresentaram distribuição normal, a comparação entre os grupos foi realizada com a ANOVA de uma via, quando uma diferença significante foi detectada procedeu-se o teste de comparaçáo múltipla de Tukey. As variáveis que não apresentaram distribuição normal foram tratadas com o teste de Kruskal-Wallis, e quando foi detectada diferença significante entre os grupos foi utilizado o teste de comparação múltipla de Dunn's.

Análise de covariância (ANCOVA) foi utilizada quando, frequência cardíaca e pressão arterial sistólica central apresentaram influência na variável dependente como covariável estatisticamente significativa. ANCOVA para medidas repetidas foi usada para comparar as medidas da tonometria 
entre os níveis de desempenho dos atletas. Os testes de ANCOVA foram seguidos pelo "posthoc” de Bonferroni. A significância estatística, para todos os testes, foi estabelecida para $\mathrm{p}<0,05$. Para análise foi utilizado o pacote estatístico SPSS versão 22 (SPSS Inc., Chicago, Illinois, USA).

\section{Resultados}

Considerando que esse estudo procurou revelar a função cardiovascular de corredores de dois diferentes níveis de desempenho atlético, comparados entre eles e entre um grupo controle, são descritas na TABELA 1 as principais características avaliadas que distinguem os grupos de corredores classificados com nível de desempenho superior, inferior e grupo controle. O GSup apresentou peso corporal e IMC reduzido em comparação aos demais grupos e menor estatura em relaçáo ao GCon, a pressão arterial sistólica periférica também foi menor em comparação aos demais grupos, já a pressão diastólica periférica foi menor apenas em relação ao grupo controle. A pressão sistólica e diastólica central foi menor para os corredores com desempenho superior em relação ao grupo controle. Confirmando a distinçáo dos corredores de acordo com a capacidade cardiopulmonar, o grupo GSup apresentou maior velocidade máxima de corrida e consequentemente um consumo máximo de oxigênio $\left(\mathrm{VO}_{2 \text { máx. }}\right)$ mais elevado.

Contudo, a respeito da classificação do desempenho pela velocidade de corrida, é importante destacar que outras variáveis ligadas ao desempenho também se diferenciaram entre os grupos, tais como distância semanal percorrida nos treinos, número e duração das sessôes semanais (FIGURA 1).
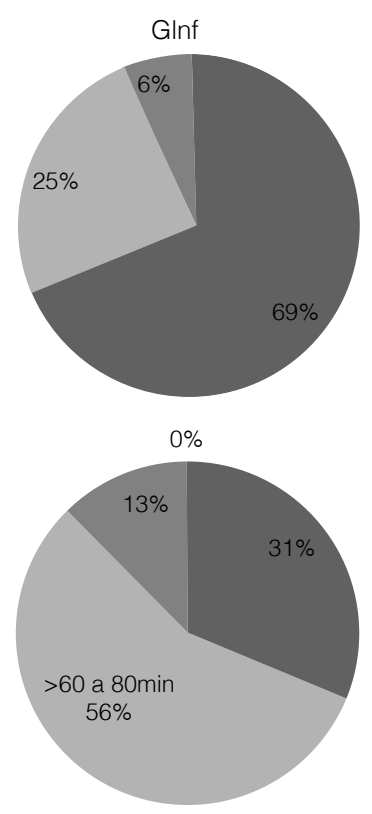

$0 \%$

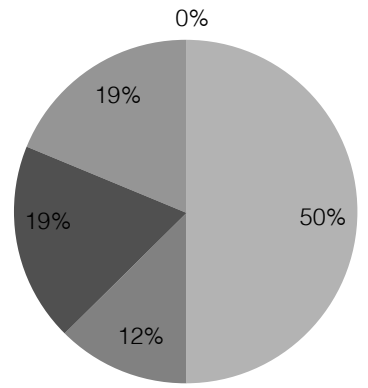

A

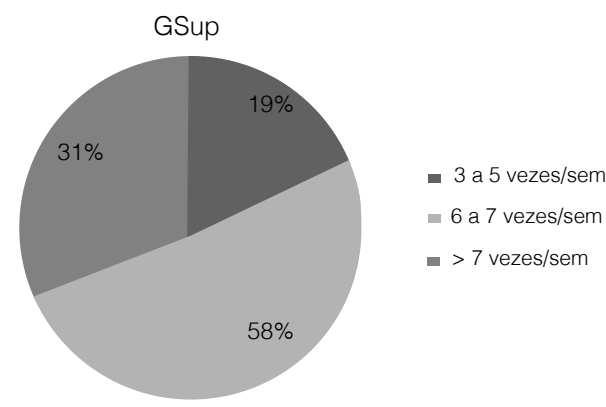

B

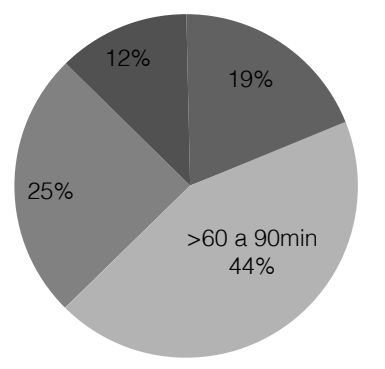

- 30 a $60 \mathrm{~min}$

- 61 a $90 \mathrm{~min}$

- 91 a 120min

- >120min
- $<40 \mathrm{~km}$

- 40 a $30 \mathrm{~km}$

- $\quad 31$ a $60 \mathrm{~km}$

- $61 \mathrm{a} 70 \mathrm{~km}$

- $>70 \mathrm{~km}$

FIGURA 1 - Descrição do regime de treinamento dos corredores. GInf - corredores com desempenho inferior; GSup - corredores com desempenho superior. A - frequência semanal; B - duração da sessão; C - distância percorrida. 
Valores expressos como média e desvio padrão. GSup: Grupo Superior; GInf: Grupo Inferior; GCon: Grupo Controle; IMC: índice de massa corporal; FC: frequência cardíaca; PASp: pressão sistólica periférica;

PADp: pressão diastólica periférica; PASc: pressão sistólica central; PADc: pressão diastólica central; Veloc. $\mathrm{VO}_{2 \text { máx. }}$; Velocidade de corrida no $\mathrm{VO}_{2 \text { máx. }}$; $\mathrm{VO}_{2 \text { máx. }}$ : Consumo máximo de oxigênio. ${ }^{*} \mathrm{P}<0,05$; GSup vs. GInf; \#P<0,05; GSup vs.GCon; ${ }^{\dagger} P<0,05$ GInf vs. GCon.
Anova de uma via; Valores expressos como média \pm desvio padrão (mediana). FCr: frequência cardíaca de repouso; PPp: pressão de pulso periférica ED: duração da ejeção (ms); DD: duração da diástole; $\mathrm{PP}_{\mathrm{p} \text {. }}$ pressão de pulso central; $\mathrm{Al}$; índice de aumento central; SEVR: índice de viabilidade subendocárdica; VOP; velocidade da onda de pulso; ${ }^{*} \mathrm{P}<0,05$ em relação a GSup vs. GInf; ${ }^{P} \mathrm{P}<0,05$ em relação GSup a VS. GCon; ${ }^{\mathrm{P}} \mathrm{P}<0,05$ em relação GInf vs.GCon.

TABELA 1 - Caracterização dos grupos classificados com nível de desempenho superior, inferior e grupo controle.

\begin{tabular}{|c|c|c|c|}
\hline Variáveis & GSup $(n=16)$ & $\operatorname{GInf}(n=16)$ & GCon $(n=16)$ \\
\hline Idade (anos) & $33 \pm 6$ & $36 \pm 5$ & $32 \pm 6$ \\
\hline Estatura $(\mathrm{cm})$ & $172 \pm 6^{\#}$ & $174 \pm 5$ & $178 \pm 5$ \\
\hline Peso corporal (kg) & $64 \pm 7^{* \#}$ & $74 \pm 5$ & $78 \pm 13$ \\
\hline IMC $\left(\mathrm{kg} / \mathrm{m}^{2}\right)$ & $21 \pm 1^{* \#}$ & $24 \pm 2$ & $23 \pm 3$ \\
\hline $\mathrm{PAS}_{\mathrm{P}}(\mathrm{mmHg})$ & $120 \pm 7(122)^{* \#}$ & $127 \pm 8(128)$ & $130 \pm 8(128)$ \\
\hline $\operatorname{PAD}_{p}(\mathrm{mmHg})$ & $68 \pm 6(68)^{\#}$ & $71 \pm 5(71)$ & $76 \pm 8(77)$ \\
\hline PASc $(\mathrm{mmHg})$ & $103 \pm 9(105)^{\#}$ & $107 \pm 6(107)^{\dagger}$ & $113 \pm 9(108)$ \\
\hline PADc $(\mathrm{mmHg})$ & $69 \pm 6(69)^{\#}$ & $72 \pm 5(72)$ & $77 \pm 8(78)$ \\
\hline Veloc. $\mathrm{VO}_{2 \operatorname{máx}}(\mathrm{km} / \mathrm{h})$ & $19 \pm 2^{* \#}$ & $14 \pm 1^{\dagger}$ & $12 \pm 1$ \\
\hline $\left.\mathrm{VO}_{2 \text { máx. }} \mathrm{ml} \cdot \mathrm{kg}^{-1} \cdot \mathrm{min}^{-1}\right)$ & $55 \pm 5^{* \#}$ & $44 \pm 2^{\dagger}$ & $38 \pm 4$ \\
\hline Anos de treino & $8 \pm 5$ & $10 \pm 6$ & - \\
\hline
\end{tabular}

Valores da hemodinâmica periférica e central para ambos os grupos são apresentados na TABELA 2. Como esperado, a frequência cardíaca de repouso foi menor em corredores com desempenho superior. A pressão de pulso periférica e central não apresentou diferenças entre os grupos. A duração da diástole foi significativamente maior no grupo de corredores com desempenho superior em relação aos demais grupos. Contudo, o índice de duração da ejeção (ED) indicador da função sistólica do ventrículo esquerdo (VE) não apresentou diferenças entre os grupos. Os indicadores de função vascular não foram diferentes entre os treinados e o controle. O AI, que indica a magnitude da reflexão da onda de pulso a partir da periferia não apresentou diferença significativa entre os grupos, bem como a velocidade de onda de pulso.

TABELA 2 - Hemodinâmica periférica e central dos corredores classificados com nível de desempenho aeróbio superior, inferior e grupo controle.

\begin{tabular}{lccc}
\hline \multicolumn{1}{c}{ Variáveis } & GSup $(\mathbf{n = 1 6})$ & GInf $(\mathbf{n = 1 6})$ & GCon $(\mathbf{n}=\mathbf{1 6})$ \\
\hline $\mathrm{FCr}(\mathrm{bpm})$ & $51,4 \pm 4(51)^{* *}$ & $55,1 \pm 9,3(55)^{\dagger}$ & $62,7 \pm 8,4(63)$ \\
\hline $\mathrm{PP}_{\mathrm{p}}(\mathrm{mmHg})$ & $51 \pm 7(51)$ & $56 \pm 9(55)$ & $52 \pm 6(51)$ \\
\hline $\mathrm{PP}_{\mathrm{C}}(\mathrm{mmHg})$ & $34 \pm 5(36)$ & $35 \pm 5(34)$ & $35 \pm 5(33)$ \\
\hline $\mathrm{DD}(\mathrm{ms})$ & $845 \pm 92(834)^{* \#}$ & $786 \pm 174(748)^{\dagger}$ & $641 \pm 128(542)$ \\
\hline $\mathrm{SEVR}(\%)$ & $218 \pm 27(218)^{* *}$ & $197 \pm 32(197)^{\dagger}$ & $167 \pm 28(167)$ \\
\hline $\mathrm{ED}(\mathrm{ms})$ & $329 \pm 27(329)$ & $332 \pm 26(332)$ & $328 \pm 18(327)$ \\
\hline $\mathrm{AI}(\%)$ & $109 \pm 12(112)$ & $109 \pm 11(107)$ & $110 \pm 19(111)$ \\
\hline $\mathrm{VOP}(\mathrm{m} / \mathrm{s})$ & $7,1 \pm 1,0(7)$ & $7,6 \pm 1,8(7)$ & $8,0 \pm 1,1(8)$ \\
\hline
\end{tabular}

$\mathrm{Na}$ TABELA 3 são apresentados ajustes adicionais das variáveis (DD, SERV, AI) pela frequência cardíaca, (VOP) e pressão arterial sistólica central. A correção pela FC se fez necessária uma vez que uma baixa frequência cardíaca pode estar associada com a melhora da diástole e do índice de perfusão. Para a VOP a correção se fez necessária, pois a pressão arterial tem uma forte influência sobre essa variável. Entretanto após as correçóes não foi encontrado diferenças entre os grupos. 
TABELA 3 - Variáveis vasculares dos corredores classificados com nível de desempenho aeróbio superior, inferior e grupo controle, corrigidas pela FC e PASc.

\begin{tabular}{lccc}
\multicolumn{1}{c}{ Variáveis } & GSup $(\mathbf{n}=\mathbf{1 6})$ & GInf $(\mathbf{n = 1 6})$ & GCon $(\mathbf{n = 1 6})$ \\
\hline DD (ms) corrigido pela FC & $758 \pm 8$ & $762 \pm 8$ & $753 \pm 8$ \\
\hline SEVR (\%) corrigido pela FC & $203 \pm 5$ & $193 \pm 4$ & $185 \pm 5$ \\
\hline AI (\%) corrigido pela FC & $105 \pm 3$ & $108 \pm 3$ & $115 \pm 3$ \\
\hline VOP $(\mathrm{m} / \mathrm{s})$ corrigido pela PASc & $7,4 \pm 1,0$ & $7,6 \pm 1,2$ & $7,8 \pm 1,2$ \\
\hline
\end{tabular}

TABELA 4 - Estrutura e função ventricular esquerda.

\begin{tabular}{lccc}
\hline \multicolumn{1}{c}{ Variáveis } & GSup $(\mathbf{n}=\mathbf{1 6})$ & GInf $(\mathbf{n}=\mathbf{1 6})$ & GCon $(\mathbf{n}=\mathbf{1 6})$ \\
\hline MVE $\left(\mathrm{g} / \mathrm{m}^{2}\right)$ & $116 \pm 12^{* *}$ & $100 \pm 18^{\dagger}$ & $86 \pm 13$ \\
\hline DC $(1 / \mathrm{min})$ & $4,8 \pm 1^{\#}$ & $5,2 \pm 1$ & $6,0 \pm 1$ \\
\hline VDF $(\mathrm{ml})$ & $131 \pm 32$ & $127 \pm 82$ & $131 \pm 25$ \\
\hline VSF $(\mathrm{ml})$ & $37 \pm 8$ & $35 \pm 12$ & $37 \pm 12$ \\
\hline VS $(\mathrm{ml})$ & $90 \pm 15$ & $91 \pm 19$ & $94 \pm 18$ \\
\hline FE $(\%)$ & $70 \pm 5$ & $71 \pm 4$ & $71 \pm 6$ \\
\hline
\end{tabular}

A MVE $\mathrm{g} / \mathrm{m}^{2}$ foi diferente entre os grupos. A massa corporal se mostrou como uma variável influenciável. A maior MVE $\mathrm{g} / \mathrm{m}^{2}$ foi mantida para os treinados após a correção pela massa corporal, o débito cardíaco demonstrou valor reduzido para o grupo Superior em relação ao grupo controle, e as demais variáveis da estrutura e função cardíaca foram semelhantes, mesmo após as correçóes.

\section{Discussão}

Este é o primeiro estudo, que temos conhecimento, a avaliar a função vascular com o uso da tonometria de aplanaçáo em uma amostra de corredores de rua, saudáveis, não maratonistas, com diferentes níveis de desempenho aeróbio, classificados em um teste cardiopulmonar. Assim, nossos resultados adquirem importância por fornecer valores hemodinâmicos pelo método não invasivo, objetivando proporcionar um melhor conhecimento sobre as propriedades dos vasos arteriais desses indivíduos. A seleção dos grupos com desempenho superior e inferior pelos valores de velocidade máxima atingida no TCPE se mostrou adequada, uma vez que se observaram diferenças no regime de treino relatado pelos grupos. O GSup relatou um regime de treinamento com maior volume, quando comparado ao GInf. Dessa forma uma maior aptidão cardiorrespiratória foi encontrada para o grupo com desempenho superior $\left(\mathrm{VO}_{2 \text { máx. }}=55 \mathrm{ml} \cdot \mathrm{kg}^{-1} \cdot \mathrm{min}^{-1}\right)$. De acordo com a literatura o $\mathrm{VO}_{2 \text { máx. }}$ tem sido uma variável de medida da aptidão cardiorrespiratória e está relacionado com a intensidade e volume de treino ${ }^{25-27}$. Em nosso estudo, mesmo tendo disponível a medida direta do $\mathrm{VO}_{2 \text { máx. }}$ considerou-se o desempenho pela velocidade máxima de corrida, por ser uma variável mais fácil de ser obtida, e essa associouse fortemente com o $\mathrm{VO}_{2 \text { máx }}(\mathrm{r}=0,93$; dados não apresentados).

Embora considerada alta para a população em geral, a aptidão cardiorrespiratória dos corredores de nível superior, selecionados para esse estudo, não atingiu patamar para considerá-los como elite desse esporte, uma vez que, valores de $\mathrm{VO}_{2 \text { máx. }}$ entre $62 \mathrm{a}$ $71 \mathrm{ml} \cdot \mathrm{kg}^{1} \cdot \mathrm{min}^{-1}$ (nível nacional) e de 75 a $78 \mathrm{ml} \cdot \mathrm{kg}^{-1}$. $\min ^{-1}$ (nível internacional) são vistos na literatura para definir corredores de alto nível ${ }^{25,26}$.

Como já esperado a FCr foi menor para o GSup, em relação aos demais grupos. Os valores pressóricos periféricos referentes à pressão arterial sistólica apresentaram-se reduzidos em relação aos demais
Estatística ANCOVA; Valores expressos como média \pm desvio padrão. Valores ajustados pela FC e PASc. Duração da diástole (DD) corrigida pela frequência cardíaca (FC); Viabilidade subendocárdica (SERV) corrigida pela frequência cardíaca (FC); Índice de incremento (Al) corrigido pela frequência cardíaca (FC); VOP corrigido pela pressão arterial sistólica central (PASc).

Anova de uma via; Valores expressos como média \pm desvio padrão. MVE $\mathrm{g} / \mathrm{m}^{2}$ : massa do ventrículo esquerdo dividida pela superfície corporal; DC: Debito cardíaco; VDF: volume diastólico final; VSF: volume sistólico final; VS: volume sistólico; FE: fração de ejeção. ${ }^{*} \mathrm{P}<0,05$ em relação a GSup vs. GInf; \#P<0,05 em relação GSup a VS. GCon; ${ }^{\dagger} P<0,05$ em relação GInf vs GCon. 
grupos, já a pressão arterial diastólica só foi diferente quando comparada ao grupo controle.

Com relação aos valores pressóricos centrais não houve diferenças entre os GSup e GInf, entretanto os dois grupos apresentaram valores reduzidos em relaçâo ao GCont mesmo após a correção para a idade. Entretanto, a função vascular medida tanto pela VOP, quanto pelo $\mathrm{AI}$, não foi diferente entre os grupos. Apenas a menor frequência cardíaca parece explicar os melhores índices pressóricos, e de perfusão miocárdica (SEVR). Por outro lado, o índice de viabilidade subendocárdica foi maior em atletas de endurance, indicando maior capacidade de extração de oxigênio pelo miocárdio ${ }^{13}$. O que se pode sugerir é que os valores pressóricos centrais estáo favorecidos para os grupos com desempenho superior e inferior uma vez que não foram encontradas diferenças entre os mesmos, porém apresentaram valores reduzidos quando comparado ao grupo controle. Esses resultados fazem sentido uma vez que o grupo estudado foi formado por indivíduos saudáveis e que independente do nível de condicionamento físico ambos se beneficiaram dos efeitos benéficos do exercício, uma vez que valores elevados da PASc estão relacionados com aumento dos riscos cardiovasculares ${ }^{28,29}$.

Em estudo recente, não se encontrou diferenças na pressão arterial de corredores recreacionais, maratonistas e de ultraendurance, na medida casual. No entanto, os mesmos autores identificaram maior valor médio da pressão arterial sistólica e pressão arterial média, quando monitoradas por $24 \mathrm{~h}$, para os corredores de ultraendurance, que executaram uma carga de $4,8 \mathrm{~h}$ de treinamento diário ${ }^{30}$. De fato, tem sido documentado elevados valores pressóricos em corredores com alto volume e intensidade de treino. Comparando um grupo controle com um grupo de maratonistas verificou-se que os atletas possuíam uma pressão arterial significativamente maior, e que a diferença na velocidade da onda de pulso desapareceu após o ajuste para pressão arterial ${ }^{31}$.

No entanto nosso grupo de corredores não se encontra com característica de alto rendimento, o que reforça que a prática de exercícios aeróbios, não extenuante, leva a melhoras significativas ao sistema cardiovascular. Em nossos resultados não foram encontradas diferenças nas curvas de PPp e de PPc, na literatura foi verificada que atletas de endurance tiveram maior pressão de pulso periférica e pressão de pulso central ${ }^{12}$. O fato de as curvas de PPp e de $\mathrm{PPc}$ não serem diferentes entre os grupos de nosso estudo pode ser um bom indicador da qualidade de seguimento arterial destes indivíduos, uma vez que estas variáveis podem contribuir como o aumento da carga cardíaca em repouso ${ }^{12}$. A rigidez arterial tem uma forte relação com a pressão arterial, sendo uma preditora de eventos cardiovasculares ${ }^{9,32}$, e sua importância tem sido realçada devido ao fato de ser uma medida integrada do impacto de diversos fatores de risco sobre a parede arterial ${ }^{33}$. Por outro lado, a prática regular de exercício aeróbio parece atenuar o enrijecimento arterial ${ }^{13-15}$. No presente estudo, a rigidez arterial foi avaliada pela mediçáo do AI através da artéria radial, e pela mediçáo da VOP carótido-femoral, em ambos os métodos não se encontrou diferença entre os grupos.

$\mathrm{O}$ índice de aumento (AI) é um indicador que permite medir a magnitude da onda de reflexão e, assim, identificar a rigidez arterial ${ }^{34}$, valores elevados do $\mathrm{AI}$ indicam precocidade da onda de reflexão da periferia (por rigidez) e/ou aumento do tempo de ejeção ventricular ${ }^{35,36}$. Fisiologicamente, em uma árvore arterial normal, a onda sistólica é refletida em todas as bifurcaçóes e regressa à raiz da aorta durante a diástole, auxiliando o enchimento coronário durante esta fase do ciclo cardíaco. Em vasos mais rígidos, verifica-se um retorno mais precoce das ondas retrógrada vindas da periferia, chegando à raiz da aorta ainda durante a sístole, aumentando a pressão sistólica central, impondo ao ventrículo esquerdo maior trabalho e comprometendo também o enchimento coronário ${ }^{37}$.

A velocidade da onda de pulso (VOP) permite avaliar a rigidez arterial através da velocidade entre a carótida-femoral, e valores elevados deste índice têm sido associados a uma maior incidência de eventos coronários e do risco cardiovascular ${ }^{38-41}$. Em nosso estudo náo foram encontradas diferenças do AI e VOP entre os grupos, mesmo após a correção do AI pela FC e da VOP pela PASc. Dessa forma os valores pressóricos mais atenuados não são explicados por redução do AI e VOP.

$\mathrm{O}$ AI é conhecido por ser afetado por uma série de variáveis incluindo a frequência cardíaca ${ }^{42}$. O grupo com desempenho superior teve uma FC de repouso menor em relação aos demais, mas ainda assim o AI não apresentou diferenças entre os grupos, mesmo após a correção para a FC.

Um estudo anterior demonstrou uma relação entre rigidez arterial e níveis diferentes de condicionamento aeróbio $\left(\mathrm{VO}_{2 \text { máx. }} 65 \mathrm{ml} \cdot \mathrm{kg}^{-1}\right.$. $\mathrm{min}^{-1}$ para indivíduos bem treinados e $\mathrm{VO}_{2 \text { máx. }}$ $49 \mathrm{ml} \cdot \mathrm{kg}^{-1} \cdot \mathrm{min}^{-1}$ para corredores ditos atletas recreacionais) a partir da artéria radial, sendo que 
o AI foi inferior para o grupo que possuía melhor desempenho físico ${ }^{13}$. Entretanto, nossos achados não foram concordantes, uma vez que não encontramos diferenças nos valores de AI entre os nossos grupos o que nos leva a acreditar que as diferenças entre os níveis de condicionamento aeróbio não foram suficientes para demonstrar tal diferença.

A PASc se apresentou como uma covariável importante para VOP, no entanto, após a correção, não foi possível encontrar diferenças entre os grupos. A literatura ressalta a influência da pressão arterial nas variaçóes da VOP, destacando que os estudos que demonstram aumento de rigidez arterial entre os corredores, chamados por ele de ultraendurance, é explicada pela maior PAS ${ }^{31}$. Por outro lado um estudo após quatro meses de intervenção aeróbia não encontrou alteração da PA central, mas verificou uma redução da $\mathrm{VOP}^{14}$, já em outro estudo foi visto que indivíduos treinados em endurance e em longo prazo exibiam menor VOP em relação ao grupo controle ${ }^{16}$.

Com relação aos valores de VOP encontrado nesse estudo $(7,1 \pm 1,0 ; 7,6 \pm 1,8 ; 8,0 \pm 1,1 \mathrm{~m} / \mathrm{s})$ nos leva a acreditar no baixo risco de rigidez arterial entre os grupos estudados, uma vez que as recomendaçóes conjuntas da Sociedade Europeia de Hipertensão e Sociedade Europeia de Cardiologia sugeriram um corte de $12 \mathrm{~m} / \mathrm{s}$ para a definição da repercussão vascular a partir da VOP aórtica. Partindo dessas recomendações, foi realizado um estudo em uma população portuguesa com 668 indivíduos saudáveis, sendo 412 homens, com idade média de 39,73 \pm 15,6 anos e pressão arterial sistólica e diastólica em torno de 125,47 $\pm 11,18$ e 75,75 $\pm 9,27 \mathrm{mmHg}$. Estes indivíduos foram submetidos a uma avaliação da VOP no período médio de dois anos e foi observado uma VOP com a média de $8,8 \pm 1,4$ $\mathrm{m} / \mathrm{s}$, sendo de $8,7 \pm 1,6 \mathrm{~m} / \mathrm{s}$ para mulheres e $8,9 \pm$ $1,5 \mathrm{~m} / \mathrm{s}$, para homens ${ }^{43}$. Dessa forma acreditamos que o efeito nulo do AI e VOP entre os grupos se justifica por se tratar de indivíduos saudáveis, que apesar da diferença no desempenho físico possuem uma saúde cardiovascular preservada.

A maior duração da diástole encontrada no grupo de corredores com desempenho superior pode ter favorecido os resultados do SEVR, indicador da perfusão coronária, que se apresentou significativamente mais elevado para esse grupo. A viabilidade subendocárdica é a razão entre a área aórtica diastólica/ área aórtica sistólica na curva de pressão aórtica. A área diastólica está associada a pressão e ao tempo de perfusão coronária, logo, com o suprimento de energia ao miocárdio, e a área sistólica está relacionada com o trabalho do coração e consequente consumo de energia ${ }^{44}$. No entanto, são necessários mais estudos com atletas de corrida em relação ao SEVR para vermos se de fato essa variável está relacionada com a perfusão coronária nesses sujeitos. De qualquer forma, como já mencionado, o índice denota maior tempo de diástole em proporção a sístole, o que poderia ser favorável para o GSup que apresentam menor pressão arterial diastólica central, o que nos parece um mecanismo compensatório favorecido pela redução da frequência cardíaca.

O grupo superior apresentou maior MVE $\mathrm{g} / \mathrm{m}^{2}$ em relação aos demais grupos, mesmo após ajuste pela massa corporal, o que reforça o efeito do treinamento na estrutura cardíaca, mas as mudanças na função cardiovascular não ficaram evidentes pelo VDF e pelo VS que foram semelhantes entre os grupos. Por outro lado, o débito cardíaco foi menor, no GSup comparado ao GCon, considerando que os exames foram realizados nos sujeitos em repouso, deitados, e a fração de ejeção não foi prejudicada, pois foram iguais para todos os grupos. É possível que no exercício máximo essa quantidade de sangue que, em repouso funciona como uma reserva dentro do coração, seja ejetada mais eficientemente nos grupos treinados, tendo em vista que eles exibiram maior massa ventricular (hipertrofia), mas isso não foi testado nesse estudo. É fato que, a importância de se ter um coração hipertrofiado seja para as situaçóes de maior demanda ${ }^{45}$, e não em repouso. Esses resultados nos levam a valorizar as adaptaçóes crônicas advindas do treinamento de corrida de rua, mas as conclusóes devem ser cautelosas quanto aos achados, uma vez que não se investigou atletas de alto nível de desempenho. Embora não se conheça claramente o limite entre os benefícios e os riscos em relação a intensidade e ao volume de exercício aeróbio na função vascular, se faz necessárias mais pesquisas com emprego de metodologia longitudinal.

Uma limitação do estudo se dá pelo fato de que, o grupo estudado se apresenta com nível de aptidão cardiorrespiratória abaixo da categoria de atletas de elite, no entanto são diferentes em relação ao regime de treino e ao $\mathrm{VO}_{2 \text { máx. }}$ o que reforça o fato da divisão dos grupos pela velocidade máxima, ter sido um critério válido. Outra limitação é o fato dos dados terem sido coletados em repouso, o que não reflete a capacidade vascular de adaptação à hemodinâmica do esforço. Dessa forma se faz necessário o desenvolvimento de estudos voltados a grupos com níveis de desempenho mais elevados, e a coleta de 
dados em situação de estresse hemodinâmico, para que possamos ter uma melhor compreensão do efeito dos diferentes níveis de treinamento aeróbio na função vascular.

De acordo com os resultados encontrados para a amostra estudada, pode-se concluir que corredores com capacidade de desempenho superior, aferida pela velocidade máxima de corrida, apresentam pressáo arterial sistólica periférica e pressão arterial sistólica central menor, acompanhada de maior tempo de duração da diástole, e menor débito cardíaco em repouso, que seus pares sedentários, mas não apresentam melhores indicadores de função vascular (AI, VOP) na mesma comparação. Esses resultados indicam que a pressão arterial dos atletas avaliados foi atenuada por menor estresse volumétrico, no período de repouso.

\title{
Agradecimentos
}

Os autores agradecem à CAPES pelo fomento de bolsa de mestrado que viabilizou o estudo, e todos os voluntários que dedicaram seu tempo e seguiram todas as orientaçôes para assegurar uma investigaçẫo bem-sucedida.

\begin{abstract}
Blood pressure and vascular function indicators of runners with different performance levels on cardiopulmonary exercise testing
\end{abstract}

The aim of this study was to compare, in the rest position, the blood pressure (BP) and the peripheral (pSBP and $\mathrm{pDBP}$ ) and central (CSBP and $\mathrm{CDBP}$ ) arterial function index in runners of different performances levels. The sample comprises 48 volunteers $20-40$ years old which 32 of them are street runners with different levels, and 16 sedentary individuals. We evaluated the cardiorespiratory fitness and the maximum speed running with cardiopulmonary exercise test (CPET). The central and peripheral pressure were performed with the applanation tonometry and the pulse wave velocity (PWV) was evaluated by Complior equipment. The echocardiogram was used to evaluate the cardiac structure. One-way ANOVA was performed considering $p<0.05$. ANCOVA was used to adjust confusion factors. Runners with higher performance, showed a lower pSBP compared to the other groups $(120 \pm 7$ vs $127 \pm 8$ vs $130 \pm 8 \mathrm{mmHg})$. The duration of diastolic was higher in runners with higher performance ( $845 \pm 92 \mathrm{~ms}$ ) compared to the runners with lower performance $(786 \pm 174)$ and controls $(641 \pm 128 \mathrm{~ms})$.The augmentation index (Al) was not different between groups $(109 \pm 21 \%, 109 \pm 11 \%, 110 \pm 1 \%)$ as well as PWV $(7.1 \pm 1 \mathrm{~ms}, 7.6 \pm 1.1 \mathrm{~ms}, 8.0 \pm 1.1 \mathrm{~ms})$. Runners with higher performance had higher left ventricular mass $\left(\mathrm{LVM} \mathrm{g} / \mathrm{m}^{2}\right)$ in relation to the others $\left(116 \pm 12 \mathrm{~g} / \mathrm{m}^{2}\right.$ vs $100 \pm 18 \mathrm{~g} / \mathrm{m}^{2}$ vs $\left.86 \pm 13 \mathrm{~g} / \mathrm{m}^{2}\right)$, and lower cardiac output compared to the control group $(4.8 \pm 1 \mathrm{vs} 6.0 \pm 11 / \mathrm{min}$.) therefore runners with higher performance had greater left ventricular mass, lower cardiac output, lower central and peripheral systolic blood pressure, and better duration of diastole their sedentary peers, but they have no better vascular function indicators in the same comparison.

KEYwords: Aerobic Exercise; Vascular Function; Arterial Stiffness; Pulse Wave Analysis.

\section{Referências}

1. Thompson PD, Buchner D, Pina IL Balady GJ, Williams MA, Berra K, et al. Exercise and physical activity in the prevention and treatment of atherosclerotic cardiovascular disease: a statement from the Council on Clinical Cardiology (Subcommittee on Exercise, Rehabilitation, and Prevention) and the Council on Nutrition, Physical Activity, and Metabolism (Subcommittee on Physical Activity). Circulation. 2003;107(24):3109-16.

2. O'Keefe JH, Schnohr P, Lavie CJ. The dose of running that best confers longevity. Heart. 2013;99(8):588-90. 
3. O'Keefe JH, Patil HR, Lavie CJ, Magalski A, Vogel RA, Mccullough PA. Potential adverse cardiovascular effects from excessive endurance exercise. Mayo Clin Proc. 2012;87(6):587-95.

4. Matos LD, Caldeira NA, Perlingeiro PS, Santos IL, Negrao CE, Azevedo IF. Cardiovascular risk and clinical factors in athletes: 10 years of evaluation. Med Sci Sports Exerc. 2011;43(6):943-50.

5. Stein R, Medeiros CM, Rosito GA, Zimerman LI, Ribeiro JP. Intrinsic sinus and atrioventricular node electrophysiologic adaptations in endurance athletes. J Am Coll Cardiol. 2002;39(6):1033-8.

6. Chapman JH. Profound sinus bradycardia in the athletic heart syndrome. J Sports Med Phys Fitness. 1982;22(1):45-8.

7. Pluim BM, Zwinderman AH, Laarse AV, Wall EE. The athlete's heart: a meta-analysis of cardiac structure and function. Circulation. 2000;101(3):336-44.

8. Heffernan KS. How healthy were the arteries of Phidippides? Clin Cardiol. 2012;35(2):65-8.

9. Vlachopoulos CD, Kardara D, Anastasakis A, Baou K, Printzios DT, Tousoulis D, et al. Arterial stiffness and wave reflections in marathon runners. Am J Hypertens. 2010;23(9):974-9.

10. Mohlenkamp S, Lehmann N, Breuckmann F, Bröcker MP, Nassenstein K, Halle M, et al. Running: the risk of coronary events: prevalence and prognostic relevance of coronary atherosclerosis in marathon runners. Eur Heart J. 2008;29(15):1903-10.

11. Neilan TG, Yoerger DM, Douglas PS, Marshall JE, Halpern EF, Lawlor D, et al. Persistent and reversible cardiac dysfunction among amateur marathon runners. Eur Heart J. 2006;27(9):1079-84.

12. Laurent P, Marenco P, Castagna O, Smulyan H, Blacher J, Safar ME. Differences in central systolic blood pressure and aortic stiffness between aerobically trained and sedentary individuals. J Am Society Hypertens. 2011;5(20):85-93.

13. Edwards DG, Lang JT. Augumentation index and systolic load are lower in competitive endurance athletes. Am J Hypertens. 2005;18(5 pt 1):679-83.

14. Hayashi K, Sugawara J, Komine H, Maeda S, Yokoi T. Effects of aerobic exercise training on stiffness of central and peripheral arteries in middle-aged sedentary men. Jpn J Physiol. 2005;55(4):23-9.

15. Currie KD, Thomas SG, Goodman JM. Effects of short-term endurance exercise training on vascular function in young males. Eur J Appl Physiol. 2009;107(2):211-8.

16. Otsuki T, Maeda S, Iemitsu M, Saito Y, Tanimura Y, Ajisaka R, et al. Relationship between arterial stiffness and athletic training programs in young adult men. J Am Society Hypertens. 2007;20(9):967-73.

17. Blair SN, Kohl HW, Barlow CE, Gibbons LW. Physical fitness and all-cause mortality in hypertensive men. Ann Med. 1991;23(3):307-12.

18. Medina FL, Lobo FS, Souza DR, Kanegusuku H, Forjaz CL. Atividade física: impacto sobre a pressão arterial. Rev Bras Hipertens. 2010;17(2):103-6.

19. Morra EA, Zaniqueli D, Rodrigues SL, Lunz W, Mill JG, Carletti L. Long-term intense resistance training in men is associated with preserved cardiac structure/function, decreased aortic stiffness, and lower central augmentation pressure. J Hypertens. 2014;32(2):286-93.

20. Jekel JF, Elmore JG, Katz DL. Introdução à medicina preventiva. In: Jekel JF, Elmore JG, Katz DL, editores. Epidemiologia, bioestatística e medicina preventiva. Porto Alegre: Artes Médicas Sul; 1999.

21. Howley TE, Basset RD, Welch GH. Criteria for maximal oxygen uptake: review and commentary. Med Sci Sports Exerc. 1995;27(9):1292-1301.

22. Aquino EM, Barreto SM, Bensenor IM, Carvalho MS, Chor D, Duncan BB, et al. Brazilian longitudinal study of adult health (ELSA-Brasil): objectives and design. Am J Epidemiol. 2012;175(4):315-24.

23. Dubois D, Dubois EF. A formula to estimate the approximate surface area if height and weight be known. Arch Intern Med. 1916;17:863-71.

24. Simone G, Devereux RB, Daniels SR, Meyer RA. Gender differences in left ventricular growth. Hypertension. 1995;26(6 pt 1):979-83.

25. Santos MT, Rodrigues AI, Greco GC, Marques AL, Terra BS, Oliveira BR. Estimated VO2max and its corresponding velocity predict performance of amateur runners. Rev Bras Cineantropom Desempenho Hum. 2012;14(2):192-201.

26. Billat VL, Lepretre PM, Heugas, AM, Laurence HM, Salim D, Koralsztein JP. Training and bioenergetics characteristics in elite male and female Kenyan runners. Med Sci Sports Exerc. 2003;35(2):297-304.

27. Esteve-Lanao J, San Juan FA, Earnest CP, Foster C, Lucia A. How do endurance runners actually train? Relationship with competition performance. Med Sci Sports Exerc. 2005;37(3):496-504.

28. Bortolotto LA. Pressão central: como interpretar na prática clínica. Rev Bras Hipertens. 2009;16(1):46-7.

29. O’Rourke MF, Staessen JA, Vlachopouls C, Duprez D, Plante, GE. Clinical applications of arterial stiffness: definitions and reference values. Am J Hypertens. 2002;15(5):426-44. 
30. Radtke T, Schmidt A, Brugger N, Schäfer D, Saner H, Wilhelm M. Ultra-endurance sports have no negative impact on indices of arterial stiffness. Eur J Appl Physiol. 2014;114(1):49-57.

31. Vlachopoulos C, Aznaouridis K, Stefanadis C. Prediction of cardiovascular events and all-cause mortality with arterial stiffness: a systematic review and meta-analysis. J Am College Cardiol. 2010;55(13):1318-27.

32. Maldonado J, Pereira T, Polónia J, Silva JÁ, Morais J, Marques M. Arterial stiffness predicts cardiovascular outcome in a low-to-moderate cardiovascular risk population: the Ediva (Estudo de Distensibilidade Vascular) Project. J Hypertens. 2011;29(4):669-75.

33. Sutton-Tyrrell K, Newman A, Simonsick EM, Havlik R, Pahor M, Lakatta E, et al. Aortic stiffness is associated with visceral adiposity in older adults enrolled in the study of health, aging, and body composition. Hypertension. 2001;38(3):429-33.

34. O'Rourke MF, Kelly RP. Wave reflection in the systemic circulation and its implications in ventricular function. J Hypertens. 1993;11(4):327-37.

35. O’Rourke M. Arterial stiffness, systolic blood pressure, and logical treatment of arterial hypertension. Hypertension. 1990 abr; 15:339-47.

36. Nichols W, O’Rourke M, Nichols WW. McDonald's blood flow in arteries: theoretical, experimental and clinical principles. London: CRC Press; 1998.

37. Carmona JP. Distensibilidade dos grandes vasos arteriais, doença hipertensiva e risco cardiovascular. Rev Port Cardiol. 2004;23(12):1551-5.

38. Gatzka CD, Cameron JD, Kingwell BA, Dart AM. Relation between coronary artery disease, aortic stiffness and left ventricular structure in a population sample. Hypertension. 1998;32(3):575-8.

39. Blacher J, Asmar R, Djane S, London GM, Safar ME. Aortic pulse wave velocity as a marker of cardiovascular risk in hypertensive patients. Hypertension. 1999;33(5):1111-7.

40. Boutouyrie P, Tropeano AI, Asmar R, Gautier I, Benetos A, Lacolley P, et al. Aortic stiffness is an independent predictor of primary coronary events in hypertensive patients: a longitudinal study. Hypertension. 2002;39(1):10-5.

41. Nichols WW. Clinical measurement of arterial stiffness obtained from noninvasive pressure waveforms. Am J Hypertens. 2005;18(1 pt 2):3-10.

42. Wilkinson IB, Maccallum H, Flint L, Cockcroft JR, Newby DE, Webb DJ. The influence of heart rate on augmentation index and central arterial pressure in humans. J Physiol. 2000;525(pt 1):263-70.

43. Pereira T, Maldonado J, Polóniad J, Alberto JS, Moraise J, Marques M. Definição de valores de referência da velocidade da onda de pulso arterial numa população portuguesa: uma sub-análise do projecto EDIVA. Rev Port Cardiol. 2011;30(9):691-8.

44. Williams B, Lacy PS, Thom SM, Cruickshank K, Stanton A, Collier D, et al. Differential impact of blood pressure-lowering drugs on central aortic pressure and clinical outcomes: principal results of the Conduit Artery Function Evaluation (CAFE) study. Circulation. 2006;113(9):1213-25.

45. Colan, SD. Mechanics of left ventricular systolic and diastolic function in physiologic hypertrophy of the athlete's heart. Cardiology Clinics. 1997;15(3):355-72.

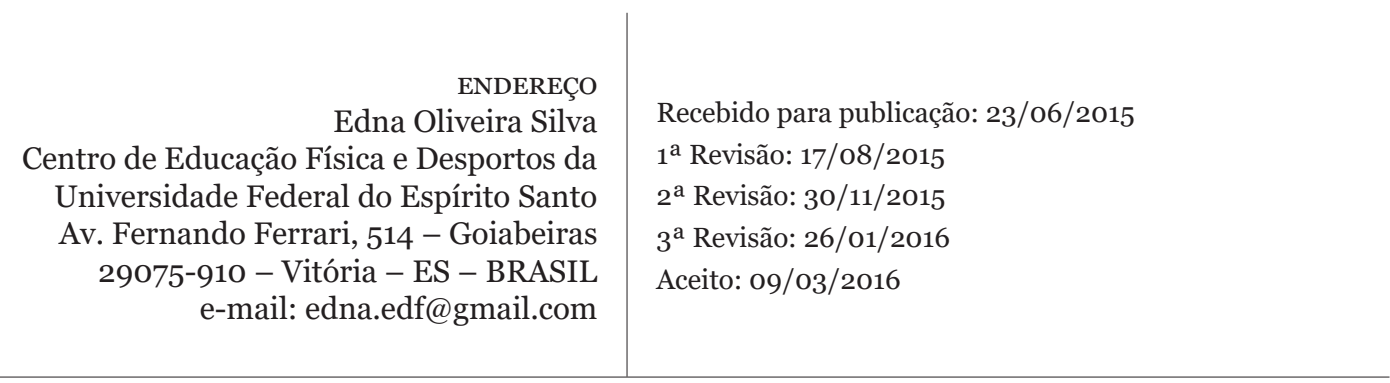

